\title{
Important Clinical Implications of Side Effects of Chemotherapy for the Brain \& Heart (Which are frequently Unrecognized) are often due to Overdose of Chemotherapy Medicine, and how to Prevent them
}

\author{
Yoshiaki Omura, M.D., Sc.D.* \\ Director of Medical Research, Heart Disease Research Foundation, USA \\ President \& Prof., International College of Acupuncture \& Electro-Therapeutic, USA \\ Adjunct Prof., Dept. of Family \& Community Medicine, New York Medical College, USA
}

According to the "Chemotherapy Side-Effects" sheet of the National Cancer Institute, which is available to the public, 18 side-effects are listed including memory problems and fatigue but not including heart problems [1]. The author often noticed that when cancer patients receiving chemotherapy treatment were offered helpful instruction the patient did not follow because the patient could not remember the instruction. Patients receiving chemotherapy treatment often complain of feeling very tired with discomfort on the heart area. For these patients, when ECGs are taken, we often found a very slight abnormality in the rising part of the $\mathrm{T}$ wave of the ECG corresponding to "Vulnerable Period for Ventricular Fibrillation." But, most cardiologists consider that the patient has no serious heart problems. When measuring the blood level of cardiac Troponin I levels in these patients, often they come back within normal limits or with slight increases. The question is what is happening to the brain and heart of these patients? Is there really no problem in the heart? When there is some evidence that only small part of heart or certain phase of ECGs has some problem, but current accepted ECG interpretation seems to have limitations of detecting them. As the consequences, no significant changes in the blood cardiac Troponin I levels and waveform and amplitude of ECG Voltage are expected. Final question is what factor of chemotherapy is worsening side effects.

To answer the first problem, with regards to memory, we examined non-invasively the amount of acetylcholine and $\beta$-amyloid (1-42) in the brain, particularly in the hippocampi. We found that acetylcholine is often very low and $\beta$-amyloid (1-42) is often abnormally increased to the level of Alzheimer's disease. Decreasing acetylcholine or increasing $\beta$-amyloid (1-42) also resembles Alzheimer's disease. We also found that asbestos is not only abnormally increased in all types of malignant tissues, but also that it is increased in the rest of the body in less concentration [2-4]. The most common asbestos is Crysotile asbestos. Often, similar to Alzheimer's patients, aluminum is also increased in the brain of some patients. These abnormal changes in the brain are the reason why the patient cannot remember certain important advice to follow. We found normal cell Telomere and Sirtuin 1 of longevity gene in the patient with the malignancy is often extremely low while the Telomere of the malignancy is very high.

To solve these brain problems, we combined 4 different methods.1) We increased normal cell Telomere significantly by using stimulation of Omura's ST36 acupuncture point or certain substances such as Haritaki, Açaí, Graviola, Turmeric, Maca, Ginger etc. (listed in the order of maximum increase in Telomere by giving optimal dose), significantly increasing normal cell Telomere not only increases Sirtuin 1 and brain circulation but it also increases circulation in the rest of the body [4]. 2) To remove asbestos and aluminum, we give Cilantro tablets to the patients. 3) In addition to maintaining good circulation and anti-viral effect, we give an optimal dose of EPA 180mg with DHA 120mg (with exception of malignant melanoma). These 3 treatments were shown to often improve short term memory. 4) Medicine may not reach the hippocampi areas. To solve this problem, we used "Selective Drug Uptake Enhancement Methods" by selectively delivering medication to the pathological area by mechanically stimulating organ representation areas of the brain and hippocampi located at the first segment of the middle finger of both hands. In addition, external application of strong (+) Qigong energy or special (+) Solar

*Corresponding author: Yoshiaki Omura, M.D., Sc.D., 800 Riverside Drive (8-I), New York, NY 10032, USA, E-mail: icaet@yahoo.com

Received January 06, 2013; Accepted January 06, 2013; Published January 09, 2013

Citation: Omura Y (2013) Important Clinical Implications of Side Effects of Chemotherapy for the Brain \& Heart (Which are frequently Unrecognized) are often due to Overdose of Chemotherapy Medicine, and how to Prevent them. J Integr Oncol 1: e105. doi:10.4172/2329-6771.1000e105

Copyright: (c) 2013 Omura Y. This is an open-access article distributed under the terms of the Creative Commons Attribution License, which permits unrestricted use, distribution, and reproduction in any medium, provided the original author and source are credited. 
Energy Stored Paper to improve circulation significantly and enhance drug uptake by applying to the area above the hippocampi.

Certain chemotherapy drugs such as Anthracycline, which has been used for many years as treatment for bone marrow related malignancies such as leukemia and lymphoma, as well as breast, uterine, ovarian and lung cancers, they are well known for producing cardiotoxicity $[3,5]$.

However, in the patient who developed severe fatigue after chemotherapy with heavy sensation in the heart area but ECGs did not show significant changes, we used a U.S. patented method of Electromagnetic Field (EMF) Resonance Phenomena between two identical substances to find abnormal concentrations of molecules in different part of the heart [2]. We found at the rising part of the $\mathrm{T}$ wave of an ECG, in spite of the very small change, the appearance of small notches or small indentations or microvolt ranges changes. This area corresponds to the electrophysiological well-known area called "Vulnerable Period for Ventricular Fibrillation." During this period, we recently found that in spite of the normal blood cardiac Troponin I levels by blood tests, the ECG recording of this area showed very high amounts of cardiac Troponin I level. Also, we often find abnormally increased calcium levels of up to $100 \%$ or higher and Manganese increased to a maximum of $100 \%$ or higher. These indicate that some of the patients, according to the standard ECG interpretations, are within normal limits, but according to our new interpretation of the ECG that it often showed significant abnormality to the specific part of the ECG. These can be causes of unexpected death normally undetected by standard ECG interpretation and no abnormal blood chemistry can be detected by standard lab tests. We are working to solve this problem and hope to report when more data becomes available. However many of those side effects are due to the repeated overdose of chemotherapy. To reduce these side effects, it is important to give individually determined optimal dose of chemotherapy agent before repeating same treatment. Before \& after the first treatment, the therapeutic effect must be evaluated by whether the treatment is really producing a desirable decrease in cancer markers and no serious worsening is taking place.However, this is rarely carried out in many leading cancer hospitals with testing often performed after a series of treatments. To simplify this evaluation procedure, we developed a"Mouth, Hand, \& Foot Writing Method" of right \& left side of body and simplified chart [2]. With this method, we can rapidly, non-invasively detect any malignancy at any part of body long before standard lab tests can detect.
We hope more studies will be performed on these potentially serious problems and that optimal individualized doses of chemotherapy agents will be given in future treatment.

\section{References}

1. "Chemotherapy Side Effects Sheets". National Cancer Institute. National Institute of Health, 10 2012. Web. 3 Jan 2013.

2. Omura Y (2013) "Asbestos: Profound Evidence for Disease Association" in the book Advancing Medicine with Food and Nutrients $\left(2^{\text {nd }}\right.$ edn $)$ Edited by Ingrid Kohlstadt. CRC Press 41: 777-798.

3. Shakir D (2009) "Chemotherapy Induced Cardiomyopathy: Pathogenesis, Monitoring and Management." Journal of Clinical Medicine Research 1: 8-12.

4. Omura $Y$, Chen $Y$, Lermand $O$, Jones $M$, Duvvi $H$, et al. (2010) "Effects of Transcutaneous Electrical Stimulation (1 pulse/sec) Through Custom-made Disposable Surface Electrodes Covering Omura's ST36 Area of Both Legs on Normal Cell Telomeres, Oncogen C-fos Ab2, Integrin a5ß1. Chlamydia Trachomatis, etc in Breast Cancer \& Alzheimer Patients". Acupuncture \& Electro-Therapeutics Research, the International Journal 35: 147-186.

5. Broder H (2008) "Chemotherapy and Cardiotoxicity". Reviews in Cardiovascular Medicine 9: 75-83. 